Medicine Updates

Faculty of medicine

October 2020,volume 3, https://muj.journals.ekb.eg

dean@med.psu.edu.eg

vice_dean_postgraduate@med.psu.edu.eg

DOI: $10.21608 / \mathrm{muj} .2020 .40893 .1022$

ISSN : 2682-2741

Submitted: $28 / 8 / 2020$

Accepted : 04/9/2020

Pages:75- 83

\title{
Role of Nitroglycerin administration through inhalation route as Nitric oxide donor in decreasing pulmonary hypertension as consequence of non-cyanotic congenital heart defects in children candidate for diagnostic cardiac catheterization
}

Nashwa Ahmed *MD, Lecturer of Anaesthesia and Surgical Intensive Care, Faculty of Medicine, Port Said University, Egypt

Aly Abd ELmohsen MD. Professor of Pediatrics, Faculty of Medicine, Alexandria University, Egypt

\begin{abstract}
$\underline{\text { Abstract }}$
Background: This work aimed to study nitroglycerin effect through inhalation route on pulmonary hemodynamics in children complaining from pulmonary hypertension associated withs noncyanotic congenital heart defects candidate for diagnostic cardiac catheterization.
\end{abstract}

Methods: This prospective study had been carried out in Cardiac Catheterization Centre at El-Shatby Pediatric Alexandria University Hospital after approval of the medical ethical committee. Each parent had received verbal and plain language written explanation of the research protocol and informed consent had been taken from their parents. Eighteen children below twelve years old complaining noncyanotic congenital heart defects and left to right shunt with pulmonary hypertension who required diagnostic cardiac catheterization had participated 
prospectively in this research. After induction of anaesthesa, systemic and pulmonary pressures had been recorded for all patients invasively. Where pulmonary vascular resistance index and systemic vascular resistance index had been determined using standard formula based on Fick's principle through computerized program. Then patients received $2.5 \mu \mathrm{g} / \mathrm{kg} / \mathrm{min}$ nitroglycerin through inhalation for $30 \mathrm{~min}$. After that all measurements had been recorded again.

Results: After nitroglycerin inhalational, mean, diastolic and systolic pulmonary pressures reduced significantly in comparison with their baseline data. Also, the PVRI had diminished from baseline data. No significate changes were recorded for systemic pressures and SVRI.

Conclusion: Nitroglycerin inhalation decreased pulmonary pressures as well as pulmonary vascular resistance index, with no effect on systemic haemodynamics nor systemic vascular resistance index in kids with pulmonary hypertension as a consequence of noncyanotic congenital heart defects.

Keywords: Nitroglycerin, Inhalation, Nitric oxide donor, Pulmonary hypertension, Catheterization .

\section{INTRODUCTION}

Congenital heart disease prevalence is about $0.8 \%$ and majority of them live to adulthood in advanced countries because of early intervention. But $90 \%$ of these children obtain suboptimal or no care in most of the third world countries (Hamid, 2012).

Patients numbers with pulmonary hypertension had increased due to improvement of diagnostic techniques and increased awareness. Majority of these patients might be enrolled in emergency or elective surgery, which had high morbidity and mortality (Tonelli \& Minai, 2014). Right heart failure as a sequalae of pulmonary hypertension is the extremely important reason for mortality and morbidity in these patients. Those patients need good peri-operative management included avoiding usage of drugs that could lower pulmonary vascular resistance, in addition to factors could precipitate pulmonary hypertensive crisis (Singh et al., 2010).

Traditional parenteral vasodilators as therapeutic treatment for pulmonary hypertension lack pulmonary vasculature selectivity and systemic pressures decreased, limiting their use. So, drugs administration through inhalation route had become more popular because of pulmonary vascular selectivity (Mansour, 2013). 
The most frequently drug administrated by inhalation route was nitric oxide, but the cost was high, require specialized equipment and its toxicity (Singh et al., 2010).

This work aimed to study nitroglycerin effect through nebulization route on pulmonary haemodynamics in children with pulmonary hypertension associated with noncyanotic congenital heart defects candidate for diagnostic cardiac catheterization.

\section{PATIENTS AND METHODS}

This prospective randomized study had been carried out in Cardiac Catheterization Centre at El-Shatby Pediatric Alexandria university hospital after approval of the medical ethical committee. Each parent had received verbal and plain language written protocol explanation of the research and informed consent had been taken from their parents. Eighteen children below twelve years old complaining of noncyanotic congenital heart defects and left to right shunt with pulmonary hypertension [defined by mean pulmonary pressure exceeds $25 \mathrm{mmHg}$ at rest or 30 $\mathrm{mmHg}$ during effort] who required diagnostic cardiac catheterization had participated prospectively in this research.

Exclusion criteria includes: Cyanotic heart disease, Coagulopathy, Eisenmenger syndrome, Primary Pulmonary Hypertension, Trisomy 21, those given sedative drugs or on inotropic drugs and also those on vasodilator treatment.

Anaesthetic technique was the same in all the patients, intravenous line was inserted after EMLA cream application. Monitors, which include ECG leads, pulse oximeter and noninvasive blood pressure were attached.

Femoral access insertion for right heart catheterization using NIH catheter Johnsen and Johnson pulmonary artery catheter for pressures measurement was done under sedation using midazolam $(0.05-0.1 \mathrm{mg} / \mathrm{kg})$ with intravenous meperidine (1-1.5 $\mathrm{mg} / \mathrm{kg}$ ) and under local anaesthesia. Haemodynamic data were collected using MACLAB $6 \mathrm{H}$, cath lab which provided the electronic means of all haemodynamic variables. While pulmonary vascular resistance index and systemic vascular resistance index were computed using standard formula based on Fick's principle through computerized program (Morgan, 2005).

At this point all patients nebulized $2.5 \mu \mathrm{g} / \mathrm{kg} / \mathrm{min}$ nitroglycerin for $30 \mathrm{~min}$ using 5-6 litre/min of oxygen using surgical nebulizer face mask. Nitroglycerin $5 \mathrm{mg}$ ( $5 \mathrm{ml}$ of $1 \mathrm{mg} / \mathrm{ml}$ ) was prepared in $20 \mathrm{ml}$ normal saline to get a mixture of $250 \mu \mathrm{g} / \mathrm{ml}$ of drug. All set of hemodynamic data were measured again after session of nebulization.

Statistics were analyzed using Paired t-test by IBM SPSS software package version 
20.0. Quantitative data were expressed by mean and standard deviation. Significance of the gained results was judged at the $5 \%$ level ( $p$ value $\leq 0.05$ ).

\section{RESULTS}

After drug nebulization did not show any significant changes in mean, diastolic and systolic systemic arterial pressures and systemic vascular resistance index. However, the mean, diastolic and systolic pulmonary pressures reduced significantly when compared with their baseline values. Also, PVRI decreased from baseline value (Table 2 and 3, Figure 1, 2 and 3). While there was no significant difference in demographic data. (Table1).

\begin{tabular}{|c|c|c|}
\hline Variables & Mean \pm SD & P -value \\
\hline Sex & $1.44 \pm .511$ & 0.637 \\
\hline Age & $6.17 \pm 3.092$ & 0.986 \\
\hline Weight & $18.00 \pm 6.088$ & 0.999 \\
\hline
\end{tabular}

Table1. Demographic data represented as Mean \pm SD

Table 2. Systemic Hemodynamic data represented as Mean \pm SD

\begin{tabular}{|c|c|c|c|}
\hline Variable & $\begin{array}{c}\text { Baseline } \\
\text { values }\end{array}$ & $\begin{array}{c}\text { After drug } \\
\text { administration }\end{array}$ & P-value \\
\hline SAP $(\mathrm{mmHg})$ & $99.72 \pm 10.73$ & $99.67 \pm 9.92$ & 0.930 \\
\hline DAP $(\mathrm{mmHg})$ & $57.72 \pm 9.38$ & $56.44 \pm 7.51$ & 0.062 \\
\hline MAP $(\mathrm{mmHg})$ & $71.61 \pm 9.51$ & $70.94 \pm 7.812$ & 0.250 \\
\hline SVRI $\left(\right.$ dyn.s $\left./ \mathrm{cm}^{5} / \mathrm{m}^{2}\right)$ & $1.860 \pm 0.391$ & $1.84 \pm 0.339$ & 0.175 \\
\hline
\end{tabular}

Where: MAP, DAP and SAP: mean, diastolic and systolic systemic arterial pressure, respectively; SVRI: systemic vascular resistance index.

Table 3. Pulmonary Hemodynamic data represented as Mean \pm SD

\begin{tabular}{|c|c|c|c|}
\hline Variable & $\begin{array}{c}\text { Baseline } \\
\text { Values }\end{array}$ & $\begin{array}{c}\text { After drug } \\
\text { administration }\end{array}$ & P- value \\
\hline SPAP $(\mathrm{mmHg})$ & $60.17 \pm 14.04$ & $51.83 \pm 13.69$ & 0.0001 \\
\hline DPAP $(\mathrm{mmHg})$ & $29.891 \pm 1.70$ & $23.39 \pm 8.95$ & 0.0001 \\
\hline MPAP $(\mathrm{mmHg})$ & $39.89 \pm 11.88$ & $32.94 \pm 10.21$ & 0.0001 \\
\hline PVRI $\left(\right.$ dyn.s $\left./ \mathrm{cm}^{5} / \mathrm{m}^{2}\right)$ & $0.63 \pm 0.238$ & $0.509 \pm 0.198$ & 0.0001 \\
\hline
\end{tabular}


Where: MPAP, DPAP and SPAP: mean, diastolic and systolic pulmonary artery pressure, respectively; PVRI: pulmonary vascular resistance index.

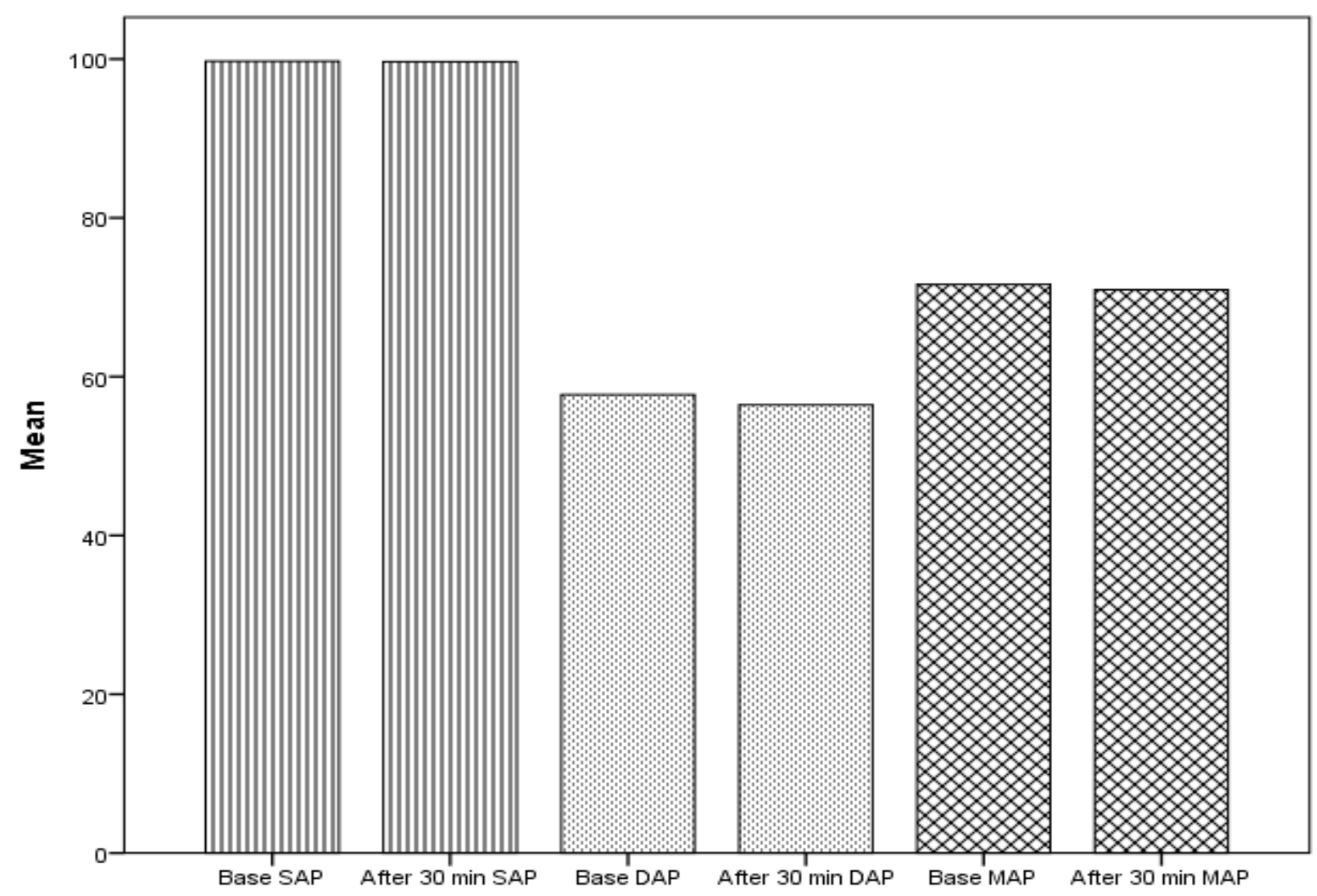

Figure 1. Systemic Hemodynamic data changes

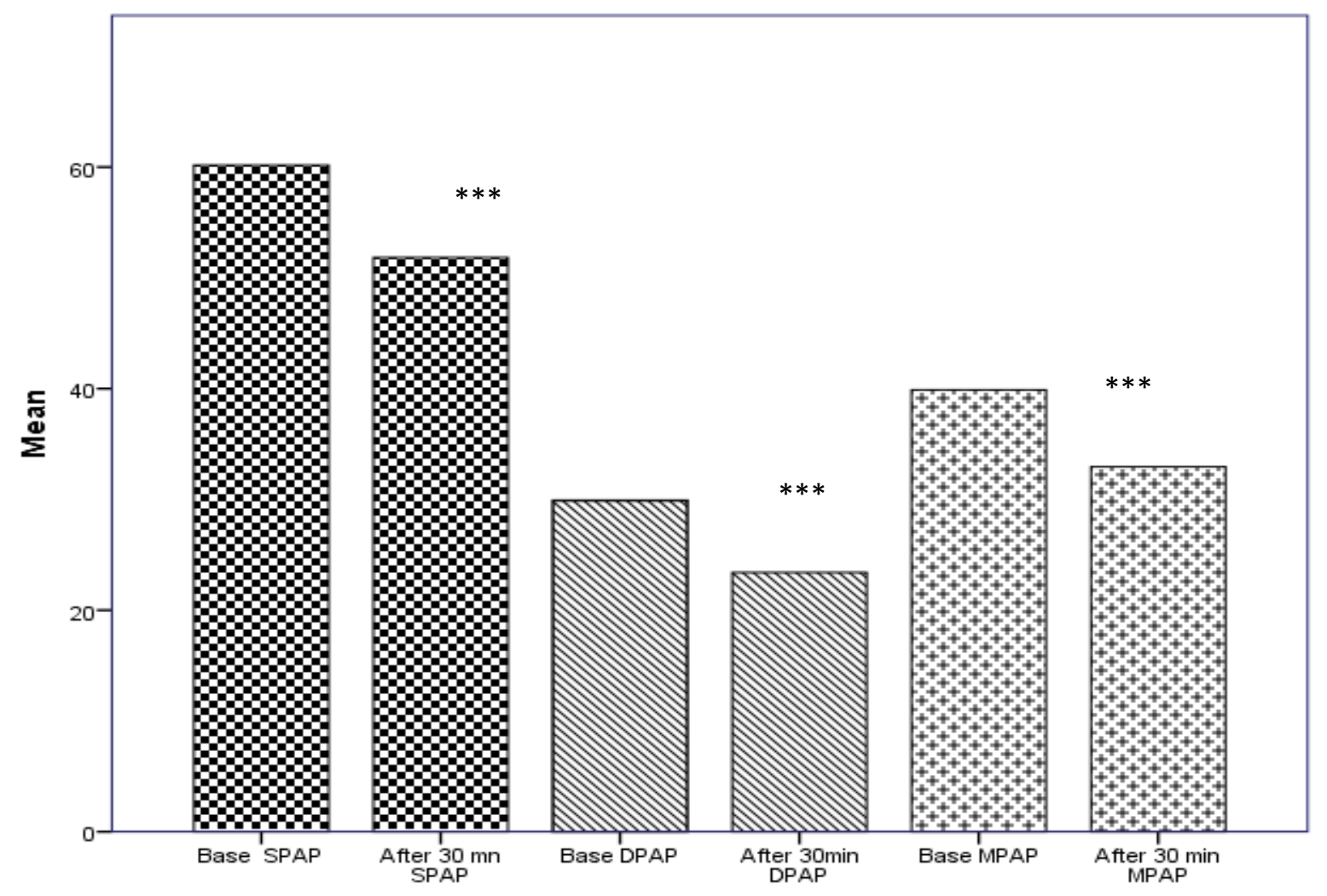


Figure 2. Pulmonary Hemodynamic data changes, $* * * \mathrm{P}=0.0001$

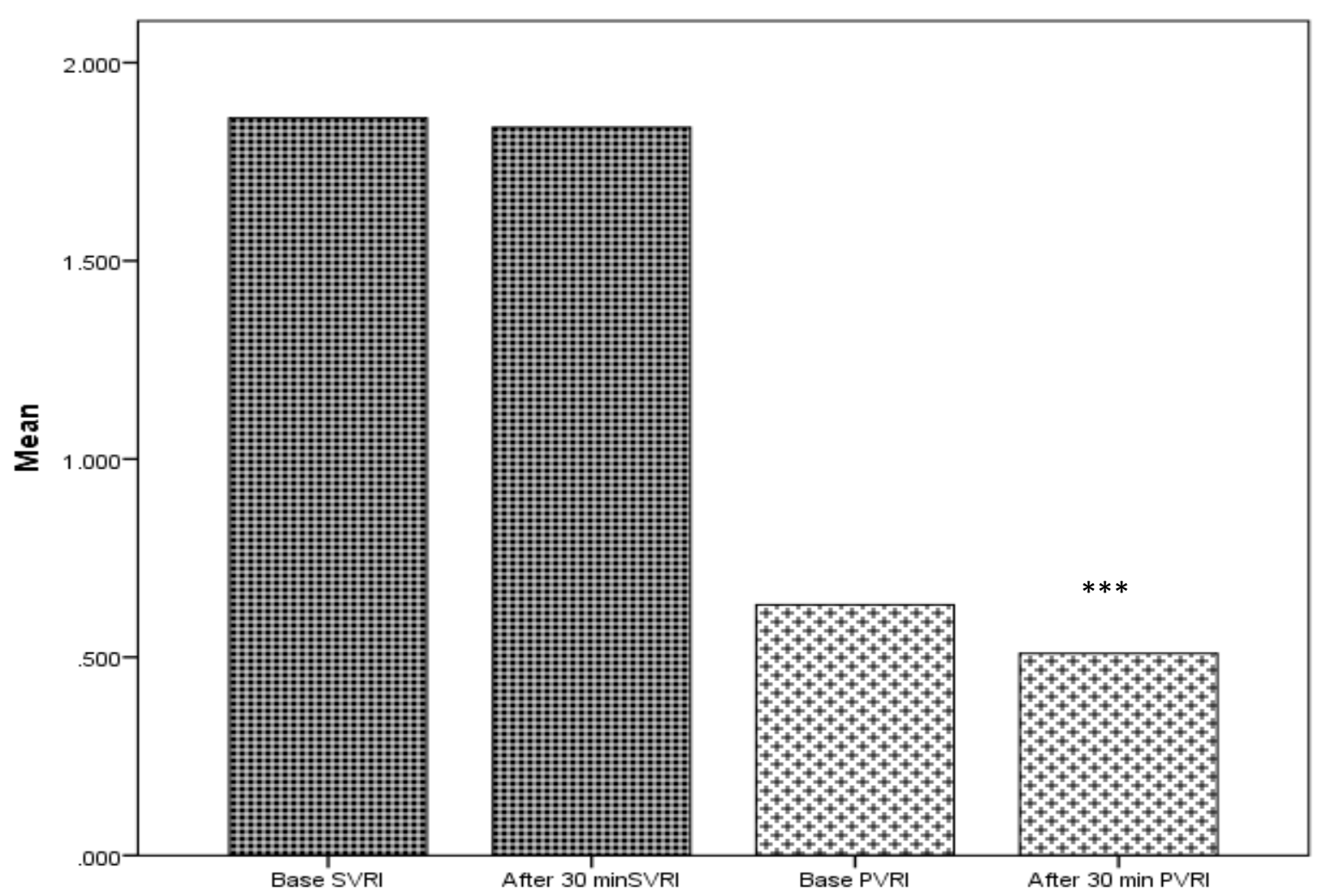

Figure 3. Pulmonary and Systemic vascular resistance index data changes, $* * \mathrm{P}=0.0001$

\section{DISCUSSION}

There had been many new studies carried on nitric oxide donor drugs via the inhalation as an alternative method of treatment in decreasing pulmonary hypertension in children with congenital heart defects such as nitroglycerin and milrinone.

Nitric oxide donor drugs could be used, at locations where facilities for inhaled nitric oxide application were not accessible mostly in circumstances where acute lessening of pulmonary a pressure was needed (Goyal et al., 2006). The results of this research revealed that nebulized nitroglycerin significantly decrease in pulmonary artery pressures and PVRI, highspoting its selectivity effect on pulmonary vasculature when administrated through the inhalation.

The same results were founded by Singh et al. (2010), they carried out a randomized clinical trial to compare nebulized milrinone and inhaled nitroglycerin acute effects on haemodynamics of systemic and pulmonary circulations in a dose of $50 \mu \mathrm{g} / \mathrm{kg}$ in children with noncyanotic congenital heart defects and pulmonary artery 
hypertension. They reported that no significant changes in systemic pressures or heart rate but there was significant reduction in pulmonary pressures.

Also found that, these drugs cause a decrease in systemic vascular resistance index and pulmonary vascular resistance index, but the reduction in systemic vascular resistance index was small in comparison with reduction in pulmonary vascular resistance index, spotlighting a more selectively effect on pulmonary circulation when given through the inhalation.

Similar observation was reported by Mansour (2013) who carried out a double blinded randomized study the effect of nitroglycerine in dose of $2.5 \mu \mathrm{g} / \mathrm{kg} / \mathrm{min}$, milrinone in dose of $5 \mathrm{mg}$, and iloprost in dose of $20 \mu \mathrm{g}$ as pulmonary vasodilators administered through the inhalation in reducing pulmonary pressures and facilitating weaning from cardiopulmonary bypass in cardiac surgical patients. Who found that there was stable systemic pressures and systemic vascular resistance index throughout the operative time while there was decrease in pulmonary vascular resistance index and in pulmonary pressures.

Moreover, Mandal et al. (2010) conducted a study using nitroglycerin in dose of 2.5 $\mu \mathrm{g} / \mathrm{kg} / \mathrm{min}$ to study comparison of the acute haemodynamic consequence of nebulized nitroglycerin, intravenous nitroglycerin and their combination with intravenous dobutamine in patients with secondary pulmonary hypertension. They reported that nebulized nitroglycerin produced selective pulmonary vasodilator without significant changes in systemic pressures and heart rate. Also, revealed that inhaled nitroglycerin decreased pulmonary blood pressures and pulmonary vascular resistance index without any significant changes in systemic pressures and systemic vascular resistance index explained by selective pulmonary vasodilator. On contrary side, they found that intravenous nitroglycerin decrease both systemic and pulmonary blood pressures as well as, pulmonary and systemic vascular resistance indexes.

Furthermore, Goyal et al. (2006) carried out a study on nebulized nitroglycerin in dose of $2.5 \mu \mathrm{g} / \mathrm{kg} / \mathrm{min}$ and its acute effects on haemodynamics of systemic and pulmonary circulations in children with pulmonary hypertension associated with congenital heart defects. Who reported that nitroglycerin nebulization significantly decreased pulmonary pressures in addition to pulmonary vascular resistance index with no effect on heart rate and systemic blood pressures.

On other hand, Bando et al. (1998) investigated effect of inhalation of nitroglycerin in dose of $2.5 \mu \mathrm{g} / \mathrm{kg} / \mathrm{min}$ on hypoxic pulmonary vasoconstriction in dogs. They revealed that nitroglycerin inhalation decreased systemic pressures, pulmonary 
pressures and pulmonary vascular resistance index but did with no effect on cardiac output. This could be due to different hemodynamics in dogs.

Furthermore, Kang et al. (2000) conducted out a research to study nitroglycerin nebulization effect in dose of $20 \mu \mathrm{g} / \mathrm{kg}$ on children with pulmonary hypertension. Who had observed that inhaled nitroglycerin decreased pulmonary artery pressure but no significantly decrease in systemic blood pressure. They explained that, by producing desired local vasodilation by liberating nitric oxide without the potential toxic gas contamination and significant systemic hypotension.

On Contrary, Nurgul et al. (2003) investigated the hemodynamic effects of nitroglycerin through inhalation in dose of $2.5 \mu \mathrm{g} / \mathrm{kg} / \mathrm{min}$ on patients with pulmonary hypertension undergoing replacement surgery of mitral valve. They reported that inhaled nitroglycerin reduced pulmonary pressures and pulmonary vascular resistance index with no effect on systemic vascular resistance index.

Based on the evidence to date, it would seem that other selective pulmonary vasodilators might be as effective as inhaled nitric oxide with less possibility for toxicity, significantly less expenses and superior easiness of application.

This study found that inhaled nitroglycerin could be used as an alternative approach for treatment in reducing pulmonary hypertension in kids with congenital heart defects, principally in clinical conditions where acute reduction of pulmonary pressure was desired and at locations where facilities for inhaled nitric oxide usage were not accessible.

\section{CONCLUSION}

It was concluded that nitroglycerin inhalation decreased pulmonary artery pressures in addition to pulmonary vascular resistance index, without affecting haemodynamics of systemic circulation in children with pulmonary hypertension as a result of noncyanotic congenital heart disease.

\section{RECOMENDATIONS}

From the present study we recommend that nitroglycerin given via the inhaled route offer a good therapeutic choice to address pulmonary artery hypertensive episodes in perioperative settings. 


\section{REFERENCES}

Bando, M., Ishii, Y., Kitamura, S., Ohno, S. (1998). Effects of inhalation of nitroglycerin on hypoxic pulmonary vasoconstriction. Respiration, 65: 63-70.

Goyal, P., Kiran, U., Chauhan, S., Juneja, R., Choudhary, M. (2006). Efficacy of nitroglycerin inhalation in reducing pulmonary arterial hypertension in children with congenital heart disease. Br J Anaesth,97:208-14.

Hamid, M. (2012). Anaesthetic Considerations for Congenital Heart Disease Patient. In: Perioperative Considerations in Cardiac Surgery. $1^{\text {st }}$ Ed. In Tech, 3: 57-74.

Kang, M., Cheng, X., Fang, G. (2000). Effect of nebulized nitroglycerin on children with ventricular septal defect and pulmonary hypertension. Journal of Zhejiang University Science, 1: 218-21.

Mandal, B., Kapoor, P., Chowdhury, U., Choudhury, M., Kiran, U. (2010). Acute hemodynamic effects of inhaled nitroglycerine, intravenous nitroglycerine and their combination with intravenous dobutamine in patients with secondary pulmonary hypertension. Annals of Cardiac Anaesthesia, 13: 138-44.

Mansour, E. (2013). Aerosolized nitroglycerin, milrinone and iloprost in cardiac surgical patients with pulmonary hypertension. Ain Shams Journal of Anesthesiology, 6:11-7.

Morgan, GE. (2005). Patient monitors. In: Clinical Anesthesiology. $3^{\text {rd }}$ Ed. New York: Lange, 6 :86-123.

Nurgul, Y., Karaca, P., Kaplan, M., Akosy, T., Kopaman, E. (2003). Effect of nitroglycerin inhalation on patients with pulmonary hypertension undergo mitral valve replacement surgery. Anesthesiology, 99: 855-8.

Singh, R., Choudhury, M., Kiran, U. (2010). Inhaled nitroglycerin versus inhaled milrinone in children with congenital heart disease suffering from pulmonary artery hypertension. Journal of Cardiothoracic and Vascular Anesthesia, 24: 797-801.

Tonelli, A. \& Minai, O. (2014). Saudi guidelines on the diagnosis and treatment of pulmonary hypertension: perioperative management in patients with pulmonary hypertension. Annals of Thoracic Medicine, 9:98-107 